\title{
Focusing Properties of Bicylindrical Acoustic Lenses
}

\author{
Tomoo KAMAKURA (The University of Electro-Communications) \\ Toshiaki NAKAMURA (National Defense Academy ) \\ kamakura@ee.uec.ac.jp \\ (Received 10 January 2007)
}

\begin{abstract}
:
This theoretical report concerns the focusing of a plane progressive acoustic wave by a bicylindrically-curved lens. It was assumed that the lens was made of polymethylmethacrylate (PMMA) and was submerged in water. The analytical approach that is based on full-wave theory began with expanding an incident plane wave into cylindrical waves to satisfy appropriate boundary conditions at an interface between the water and the lens. The continuity conditions of displacement and stress at the interface may determine the generation of longitudinal and shear waves in the lens. Lens-aperture effects on focused field performance were taken into account by confining the incident wave to the region within the aperture. The Rayleigh integral associated with the particle velocity on the exit interface of the lens enabled us to evaluate the entire sound field behind the lens. Numerical examples demonstrated no significant influence on the fields near the focus, even when the shear wave generation was included in the theory. Wave diffraction due to a finite lens aperture, however, became a major effective factor in beam focusing.
\end{abstract}

Classification: Physical acoustics, Wave propagation

Keyword: Acoustic lens, Bicylindrical lens, Focusing, Resolution

\section{Introduction}

Acoustic lenses are widely used in ultrasonic-imaging systems to display high spatial resolution images and to allow ultrasounic equipment in industrial technology to reach a high acoustic power at a focal point. The lens, ordinarily made of plastic or solid material, conveys sound at a higher speed than the surrounding medium, e.g., water. Therefore, the lens configuration should be concave.

Geometric ray theory is a powerful predictor like optics and provides a first approximation in evaluating lens performance. The ray model, however, breaks down in principle near the focus, where wave diffrac- tion becomes a significant factor in sound field formation. Additionally, it does not take account of the shear wave that may be generated at the interface between the lens and surrounding fluid and may have potential effects on field profiles. In contrast, wave acoustics can accurately analyze fields, even for mode conversion, although some tedious numerical computation is needed.

This study discusses problems of a single lens whose shape is bicylindrical and which is made of polymethylmethacrylate (PMMA). The lens is submerged in water. The principle concerns are twofold. The first is to investigate the effects of shear wave 
generation on focusing. To this end, we solve wave equations, subject to appropriate boundary conditions, for the general situation where a plane wave is incident obliquely on the lens. This situation includes the generation not only of longitudinal but also of shear waves through the process of mode conversion at the boundary. To predict the focused field behind the lens, we use the Rayleigh integral and the particle velocity on the exit surface of the lens. Several researchers have pointed out that the mode conversion is of no importance in lens performance and that only slight differences occur even if the presence of shear waves is accounted for ${ }^{1-4)}$. If their opinion is correct, it considerably simplifies computational problems ${ }^{5-7}$. Unfortunately, however, their analyses still lack quantitative evaluation. Some numerical examples in this report demonstrate that sound pressure profiles near the focus are less affected by the shear wave generation like several researchers' speculation.

The second aim of this study is to clarify the effect of lens aperture on field formation. An actual lens has a finite aperture size that determines the focal length and pressure peak at the focus. In some cases, various lens aberrations, especially cylindrical aberration, may crucially affect lens performance. Numerical simulations show that decreasing the aperture size widens the beam pattern at the focus. It does not necessarily, however, reduce the pressure peak in a smooth fashion. Other interesting findings are presented in the present demonstrations.

\section{Theory for a bicylindrical lens}

Let us imagine that a plane wave with infinite length in the $y$ axis perpendicular to the present paper surface is obliquely incident on a cylindrically curved acoustic lens, as shown in Fig. 1. The lens, made of solid material with density $\rho_{0}$, has radius $d$ and a finite aperture of $2 a$. The longitudinal and shear wave speeds in the lens are $c_{l}$ and $c_{t}$, respectively. The lens

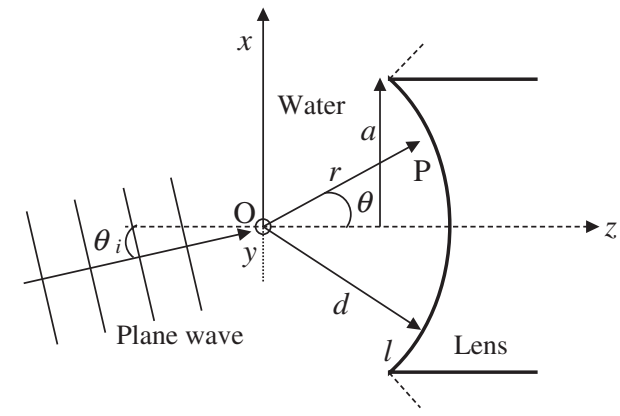

Fig. 1 Geometry of a cylindrical lens with infinite length in the $y$ axis.

is surrounded by water, whose density is $\rho$ and which has the sound speed $c$. An additional assumption is that all the sound absorptions in the lens and water can be neglected for ad hoc simplicity. We introduce the velocity potential $\phi_{i}$ with a unity amplitude to express the incident plane wave that is propagating in the $z$ direction at some angle $\theta_{i}$. In this case, $\phi_{i}$ is given by

$$
\phi_{i}=e^{-j k r \cos \left(\theta-\theta_{i}\right)},
$$

where $k=\omega / c$ is the wavenumber and $\omega$ is the angular frequency of the wave. $r$ in eq. (1) is the distance between the cylinder's origin $\mathrm{O}$ and a receiving point $\mathrm{P}$, then $\sqrt{z^{2}+x^{2}}$. Additionally, the point $\mathrm{P}$ makes an angle $\theta\left(=\tan ^{-1}(x / z)\right)$ with the $z$ axis.

Incidentally, the following identical equation holds for arbitrary variables $\xi$ and $\varphi$ :

$$
e^{-j \xi \cos \varphi}=\sum_{n=-\infty}^{\infty}(-j)^{n} J_{n}(\xi) e^{-j n \varphi}
$$

with $J_{n}(\cdot)$ being the Bessel function. Now, suppose that the incident waves inside the aperture are all gathered by the lens and the waves outside the aperture are absorbed perfectly without any reflection. This means that $\phi_{i}$ must satisfy $\exp \left[-j k d \cos \left(\theta-\theta_{i}\right)\right]$ for $|x| \leq a$ and vanish for $|x|>a$. Then 


$$
\begin{aligned}
\phi_{i} & =\sum_{n=-\infty}^{\infty} a_{n} H_{n}^{(2)}(k d) e^{-j n \theta} \\
& =\left\{\begin{array}{cc}
\sum_{n=-\infty}^{\infty}(-j)^{n} J_{n}(k d) e^{-j n\left(\theta-\theta_{i}\right)} & -\theta_{0} \leq \theta \leq \theta_{0} \\
0 & \text { otherwise, }
\end{array}\right.
\end{aligned}
$$

where $H_{n}^{(2)}(\cdot)$ is the Hankel function of the second kind, and $\theta_{0}$ is the opening angle of the inlet aperture that is given by $\sin ^{-1}(a / d)$. The arbitray coefficients $a_{n}$ in eq. (3) are determined using the orthogonality property of trigonometric functions. Multiplying both sides by $\exp [j m \theta]$ and integrating the resultant equation with respect to $\theta$ over $-\pi \leq \theta \leq \pi$ result in

$$
\begin{aligned}
a_{n} & =\frac{1}{H_{n}^{(2)}(k d)} \sum_{m=-\infty}^{\infty}(-j)^{m} e^{j m \theta_{i}} J_{m}(k d) \\
& \times \frac{\sin \left[(n-m) \theta_{0}\right]}{n-m} .
\end{aligned}
$$

When the incident wave reaches the inlet interface $l$ where the properties of the medium or acoustic impedance change abruptly, we can expect both a reflection and transmission of the wave to occur. These basic phenomena generate at least three waves; i.e., a reflected wave, which travels back to the water, and longitudinal and shear waves, which travel forward in the lens as elastic bulk waves. In the same manner as in the previous report ${ }^{8)}$, the analytical solutions of the three waves are obtained. For the reflected wave, the velocity potential $\phi_{r}$ takes the form:

$$
\phi_{r}=\sum_{n=-\infty}^{\infty} a_{n} A_{n} H_{n}^{(1)}(k r) e^{-j n \theta} \quad(r \leq d),
$$

where $H_{n}^{(1)}(\cdot)$ is the Hankel function of the first kind. $A_{n}$ will be determined explicitly by boundary conditions a short time later.

The next problem is to derive wave potentials for the longitudinal and shear waves in the lens. The displacement vector $\boldsymbol{u}$ in a solid is given by

$$
\boldsymbol{u}=\nabla \psi+\nabla \times \boldsymbol{A}
$$

The vector potential $\boldsymbol{A}$ is always solenoidal; i.e., $\nabla \cdot \boldsymbol{A}=0$. In this case, only the component $A_{y}$ in the $y$ axis is essential for the displacement generation. Another scalar potential $\psi$ that describes the longitudinal wave is also generated, and these two components take the following forms:

$$
\begin{aligned}
& \psi=\sum_{n=-\infty}^{\infty} a_{n} B_{n} H_{n}^{(2)}\left(k_{l} r\right) e^{-j n \theta} \quad(r \geq d), \\
& A_{y}=\sum_{n=-\infty}^{\infty} a_{n} C_{n} H_{n}^{(2)}\left(k_{t} r\right) \frac{d}{d \theta} e^{-j n \theta} \quad(r \geq d) .
\end{aligned}
$$

Here, $k_{l}=\omega / c_{l}$ and $k_{t}=\omega / c_{t}$ are the wavenumbers of the longitudinal and shear waves, respectively. Furthermore, the coefficients $B_{n}$ and $C_{n}$ with the participation of $A_{n}$ are completely determined by specifying three appropriate boundary conditions on the inlet interface $l:$ one is the continuity of the components of the particle velocity normal to the interface, and the other is the continuity of the stress component normal to the interface. The remaining condition is obtained by speculating that the tangential component of the stress vanishes because of the water being inviscid. Thus, we can determine $A_{n}, B_{n}$, and $C_{n}$ completely from the three boundary conditions, although some perseverance is needed to derive the solutions. To help the readers, in Appendix A we provide the coefficients $A_{n}$ to $C_{n}$ and the consequently obtained components $u_{r}$ and $u_{\theta}$.

An actual lens has an exit interface $l_{2}$, as shown in

Fig. 2. When waves travel through the lens and reach

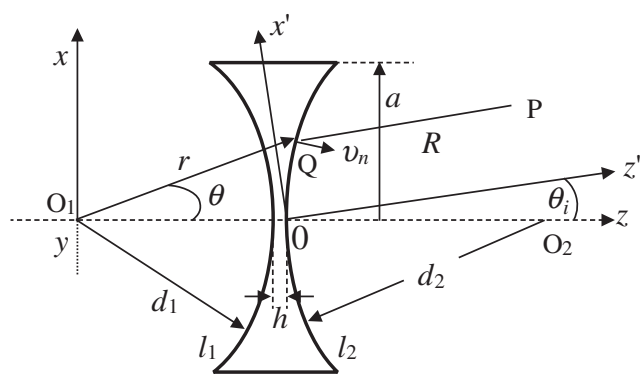

Fig. 2 Geometry of a bicylindrically focused lens. 
the exit interface, they are partially reflected owing to the change in acoustic impedance. The thus reflected waves travel back to the inlet, and wave reflection occurs again at $l_{1}$. As a first approximation, we neglect such multiple reflections among the inlet, exit, and lateral faces of the lens because the non-planar configulation of the bicylindrical interfaces is likely to weaken any reflections. According to our recent investigation, the transmission coefficient of a plane wave for a plano-concave lens is formulated using a simple model based on geometrical acoustics, even for relatively wide-angle opening ${ }^{9)}$ :

$$
\begin{aligned}
& v_{n}=T_{v}(\boldsymbol{v} \cdot \boldsymbol{n}), \\
& T_{v}=\frac{2 Z_{l}}{Z_{w}+Z_{l}},
\end{aligned}
$$

where $v_{n}$ is the normal component of the particle velocity on the interface, and $v$ is the particle velocity when the interface is hypothetically absent, expressed as

$$
\boldsymbol{v}=j \omega\left(u_{r} \boldsymbol{e}_{r}+u_{\theta} \boldsymbol{e}_{\theta}\right),
$$

where $\boldsymbol{e}_{r}$ and $\boldsymbol{e}_{\theta}$ are unit vectors along the positive $r$ and $\theta$ directions. Furthermore, $\boldsymbol{n}$ is the outer unit normal vector on $l_{2}, T_{v}$ is the transmission coefficient of the particle velocity at normal incidence on a plane boundary, and the specific acoustics impedances for the longitudinal wave is $Z_{w}$ in water, and $Z_{l}$ in the lens. To evaluate the total sound field behind the lens, we employ the two-dimentional Green function, which has the form with the Hankel function of order zero. Namely,

$$
\phi=-j \frac{1}{2} \int_{l_{2}} v_{n} H_{0}^{(2)}(k R) d s .
$$

Here $R$ is the distance between a source point $\mathrm{Q}\left(x_{0}, z_{0}\right)$ on $l_{2}$ and a receiving point $\mathrm{P}(x, z)$ and is expressed as $R=\sqrt{\left(x-x_{0}\right)^{2}+\left(z-z_{0}\right)^{2}}$ from Fig. 2 .
In addition, $d s$ in eq. (12) is the length element on $l_{2}$ and is given by $d s=d_{2} d \theta_{2}$. Therefore, eq. (12) yields

$$
\phi=-j \frac{d_{2}}{2} \int_{-\theta_{m}}^{\theta_{m}} v_{n} H_{0}^{(2)}(k R) d \theta_{2}
$$

where $\theta_{m}$ is the opening angle of the exit aperture, being given by $\sin ^{-1}\left(a / d_{2}\right)$.

\section{Numerical examples}

To know how much the shear wave and lens parameters such as the aperture radius affect focused fields behind the lens, we now demonstrate lens performance numerically. Just like the previous report ${ }^{8)}$, we choose acrylic resin or polymethylmethacrylate (PMMA) as the lens material, listing the physical quantities of PMMA in Table 1. The wave speed and sound absorption were measured at a frequency of $1 \mathrm{MHz}$ and at room temperature using longitudinal and shearwave transducers in direct contact with PMMA plates of different thicknesses in a through-transmission experiment. These values were almost same as data in the literature ${ }^{10-12)}$. The lens radii $d_{1}$ and $d_{2}$ may be changed variously. However, we confined ourselves to preparing two lenses with different radii but almost the same focal lengths. One of them is $d_{1}=60 \mathrm{~mm}$ and $d_{2}=60 \mathrm{~mm}$ (hereinafter simply called the "60/60-mm lens"), and the other is $d_{1}=100 \mathrm{~mm}$ and $d_{2}=35.5 \mathrm{~mm}$ (the "100/35.5-mm lens"). The thickness $h$ is the same,

Table 1 Physical quantities of a PMMA lens. Subscript "l" refers to longitudinal waves, and " $s$ " refers to shear waves. The sound-absorption coefficients and speeds are measured at $1 \mathrm{MHz}$ and at room temperature.

\begin{tabular}{l|c}
\hline Lens density $\rho_{0}\left[\mathrm{~kg} / \mathrm{m}^{3}\right]$ & 1180 \\
Sound velocity $c_{l}[\mathrm{~m} / \mathrm{s}]$ & 2760 \\
Sound velocity $c_{s}[\mathrm{~m} / \mathrm{s}]$ & 1340 \\
Absorption coefficient $\alpha_{l}[$ neper $/ \mathrm{m}]$ & 15 \\
Absorption coefficient $\alpha_{s}[$ neper $/ \mathrm{m}]$ & 39 \\
\hline Water density $\rho\left[\mathrm{kg} / \mathrm{m}^{3}\right]$ & 998 \\
Sound velocity $c[\mathrm{~m} / \mathrm{s}]$ & 1483 \\
Sound absorption & neglected \\
\hline
\end{tabular}


$2 \mathrm{~mm}$, for the two lenses. Because of a relatively great sound absorption in the lens, we must modify our theory. Fortunately, we can accomplish this modification by approximating the wave numbers as $k_{l}=\omega / c_{l}-j \alpha_{l}$ and $k_{t}=\omega / c_{t}-j \alpha_{t}$ in place of $k_{l}=\omega / c_{l}$ and $k_{t}=\omega / c_{t}$ eqs. (7) and (8).

Fig. 3 shows pressure-amplitude profiles along the beam axis when an incoming plane wave is normally incident on the $60 / 60-\mathrm{mm}$ lens $\left(\theta_{i}=0\right)$. To examine only the effect of shear waves on the fields, the sound absorptions in the lens are neglected in the first stage; i.e., $\alpha_{l}=\alpha_{t}=0$. Additionally, the lens aperture radius $a$ is assigned to be $37.5-\mathrm{mm}$ under the constraint that the incident waves would be confined within the critical angle $\theta_{c}$. Incidentally, the angle, defined as $\theta_{c}=\sin ^{-1}\left(c / c_{l}\right)$, has a value of $32.5^{\circ}$, so that $a$ is greater than $d_{1} \sin \theta_{c}=32.2-\mathrm{mm}$ beam. The abscissa denotes the axial distance beginning at the lens center 0, as indicated in Fig. 2. The ordinate denotes the pressure amplitude relative to the incoming pressure amplitude of $|p|=\left|j \omega \rho \phi_{i}\right|=\omega \rho$. This amplitude level corresponds to the focusing gain.

It is apparent from these data that the wave is focused by the effect of the lens. The pressure increas-

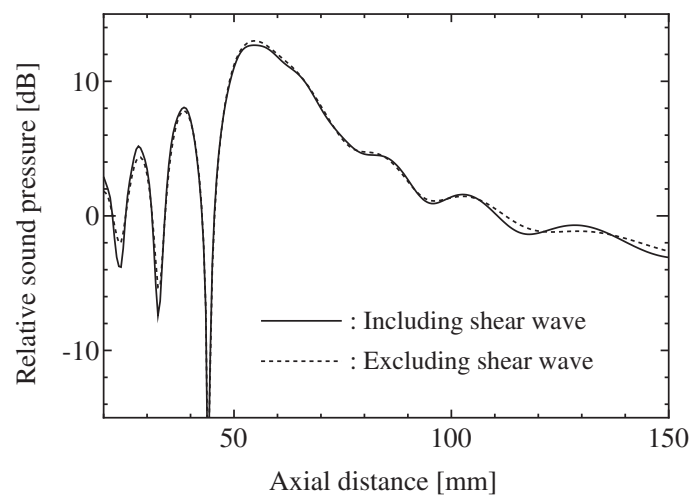

Fig. 3 On-axis pressure curves behind the $60 / 60-\mathrm{mm}$ lens with aperture radius $a=37.5 \mathrm{~mm}$ (solid curve). A 1$\mathrm{MHz}$ plane wave is impinging normally on the lens $\left(\theta_{i}=0\right)$. Sound absorption in the lens is not taken into account. The data are plotted in dots for the neglected generation of the shear wave. es in amplitude with peaks and dips during propagation, attaining a maximum amplitude of about $13 \mathrm{~dB}$ at the focus near $55 \mathrm{~mm}$ and then decreasing gradually. In the figure, the propagation curve is shown in dots when the shear wave generation is neglected by setting all the coefficients $C_{n}$ and shear terms to zero. Interestingly, the two curves overlap almost over a long range, from $20 \mathrm{~mm}$ to $150 \mathrm{~mm}$, that includes the focus. To be precise, there is some difference in the pre-focal and post-focal regions, but since this difference is slight, we may actually neglect the shear-wave generation in the design of lenses even when the aperture is too large to include the critical angle.

A similar numerical simulation is executed for the 100/35.5-mm lens. The results are shown in Fig. 4. The aperure radius is changed to $a=30 \mathrm{~mm}$, being smaller than $d_{1} \sin \theta_{c}=53.7 \mathrm{~mm}$. Therefore the mode conversion seems not to occur actively. In fact, the two pressure curves have no noticeable difference in amplitude except for the post-focal region greater than $30 \mathrm{~mm}$ from the focus. Due to there being less aberration, the 100/35.5-mm lens leads to the generation of a high acoustic pressure compared with the $60 / 60-\mathrm{mm}$ lens, although the aperture of the former lens is smaller than that of the latter.

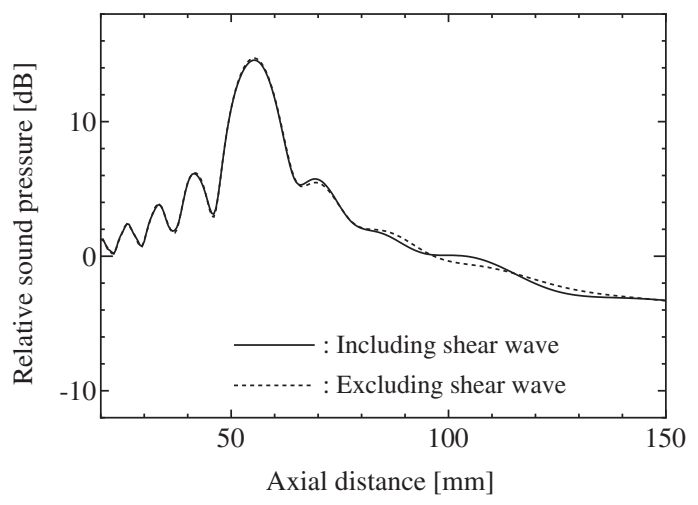

Fig. 4 On-axis pressure curves behind the 100/35.5-mm lens with aperture radius $a=30 \mathrm{~mm}$ (solid curve). A 1$\mathrm{MHz}$ plane wave is impinging normally on the lens $\left(\theta_{i}=0\right)$. The data are plotted in dots for the neglected generation of the shear wave. 
Fig. 5 shows pressure curves for different angles of the wave incidence from $\theta_{i}=0$ to $15^{\circ}$. The abscissa denotes the distance from the starting point 0 along the new axis $z^{\prime}$. The new Cartesian coordinates $\left(x^{\prime}, z^{\prime}\right)$ is related with the old ones $(x, z)$ in a familiar form:

$$
\left.\begin{array}{c}
x=x^{\prime} \cos \theta_{i}+z^{\prime} \sin \theta_{i} \\
z=-x^{\prime} \sin \theta_{i}+z^{\prime} \cos \theta_{i}
\end{array}\right\} .
$$

Strictly speaking, the beam axis with an amplitude peak is slightly shifted from the $z^{\prime}$ axis in a positive

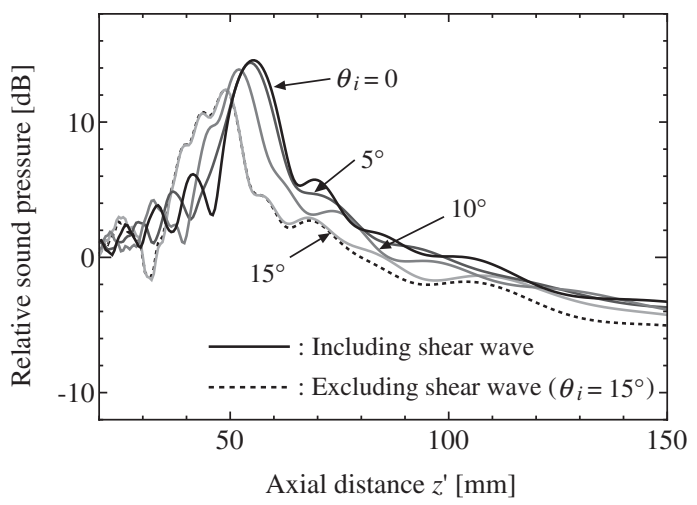

Fig. 5 Pressure curves along the $z^{\prime}$ axis for the 100/35.5-mm lens with aperture radius $a=30 \mathrm{~mm}$. A $1-\mathrm{MHz}$ plane wave is impinging obliquely on the lens from $\theta_{i}=0$ to $15^{\circ}$. The curve indicating the neglected generation of the shear wave is plotted in dots for $\theta_{i}=15^{\circ}$ only. direction of the $x^{\prime}$ axis because of the finite lens thickness $h=2 \mathrm{~mm}$. The shift is given approximately by $\Delta x^{\prime}=h \times\left(c_{l} / c\right) \sin \theta_{i}$ for small incident angles and is generally quite small. For example, the shift takes a value of only $0.66 \mathrm{~mm}$ for $\theta_{i}=10^{\circ}$. Actually, the larger the incident angle becomes, the more the amplitude peak moves towords the lens, and the more the amplitde decreases. These tendencies of the peak movement and amplitude reduction are the same as the fields in a single lens system with a spherically curved configuration $^{8}$. An additional curve in dots indicates the data from neglecting the generation of the shear wave. Importantly, the shear-wave generation somewhat influences the pressure profile in comparison with the normal-incidence case. However, the profile around the focus is unchanged whether the shear wave is included or excluded.

Beam patterns or pressure amplitude profiles along the lines perpendicular to the $z^{\prime}$ axis are shown in Fig. 6, where the incident angle is changed from $\theta_{i}=0$ to $15^{\circ}$ every $5^{\circ}$. Observational positions are determined by amplitude peaks in Fig. 5 ; i.e., $z^{\prime}=55.5 \mathrm{~mm}$ for $\theta_{i}=0, z^{\prime}=54.7 \mathrm{~mm}$ for $\theta_{i}=5^{\circ}, z^{\prime}=51.9 \mathrm{~mm}$ for $\theta_{i}=10^{\circ}$, and $z^{\prime}=48.8 \mathrm{~mm}$ for $\theta_{i}=15^{\circ}$. The Rayleigh's

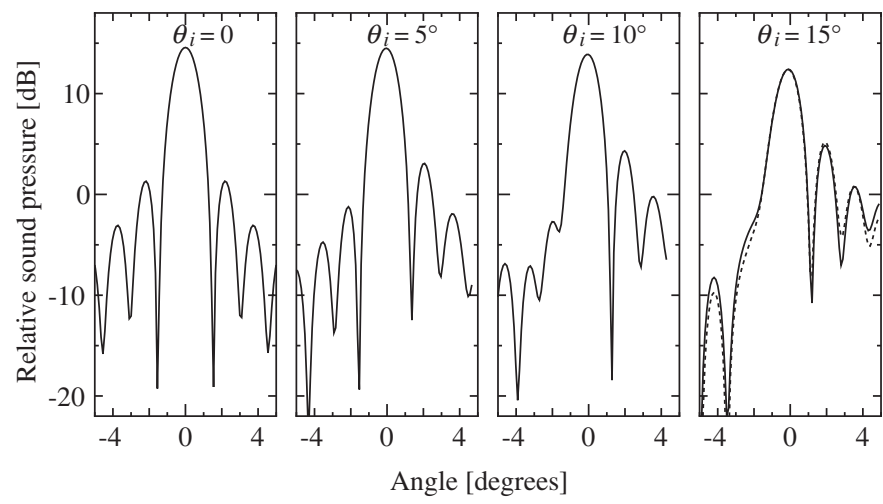

Fig. 6 Beam patterns at various incident angles from 0 to $15^{\circ}$ for the 100/35.5$\mathrm{mm}$ lens with aperture radius $a=30 \mathrm{~mm}$. The receiving points on the beam axis are chosen at the pressure peaks in Fig. 5; i.e., $z^{\prime}=55.5 \mathrm{~mm}$ for $\theta_{i}=0, z^{\prime}=54.7 \mathrm{~mm}$ for $\theta_{i}=5^{\circ}, z^{\prime}=51.9 \mathrm{~mm}$ for $\theta_{i}=10^{\circ}$, and $z^{\prime}=48.8$ $\mathrm{mm}$ for $\theta_{i}=15^{\circ}$. The curve indicating the neglected generation of the shear wave is plotted in dots for $\theta_{i}=15^{\circ}$ only. 
criterion $^{13)}$ that is regarded as a measure of image resolution and is defined as $\Delta \theta=\lambda / 2 a$ for a long and narrow aperture or slit ( $\lambda$ is the wavelength) becomes $1.4^{\circ}$ by substituting $\lambda=1.48 \mathrm{~mm}$ and $a=30 \mathrm{~mm}$. Since the present numerical data of the angle resolution indicate about $1.5^{\circ}$, which is a little larger than the theoretical value that is up to at least $10^{\circ}$, this single lens system may serve roughly as the upper limit of the angle resolution. At $\theta_{i}=15^{\circ}$, however, the beam seems to broaden. Furthermore, the amplitude peak is decreased by several decibels in comparison with those at other angles, and the patterns of the sidelobes are pronouncedly asymmetric with respect to the beam axis. The pressure levels of the sidelobes are increased as well. A dotted line in the figure denotes the data for $\theta_{i}=15^{\circ}$ only for the case assuming that the shear wave is absent. Like in Fig. 5, the generation of the shear wave hardly influences the focused field in the paraxial region within $\pm 2^{\circ}$.

It is expected that decreasing the aperture size would move the acoustic focus towards the lens because wave diffraction appears prominently in small apertures. Fig. 7 shows axial pressure profiles for normal incidence to the $60 / 60$-mm lens. The aperture radius is changed from $a=50 \mathrm{~mm}$ to $15 \mathrm{~mm}$. Actually,

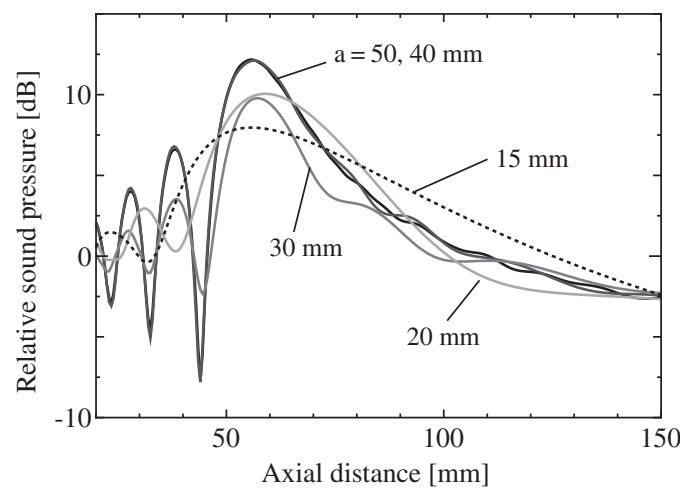

Fig. 7 Pressure-amplitude profiles along the axis by varing the aperture radius for the $60 / 60-\mathrm{mm}$ lens. Plane waves are normally incident on the lens. Sound absorptions in the lens are included in the data. decreasing the aperture makes the pressure peak low and moves the focus toward the lens, indicated, for example, by comparing the data between the $30-\mathrm{mm}$ radius aperture and the $15-\mathrm{mm}$ one. In detail, we note that first the focus moves somewhat away from the lens and that it then slightly approaches the lens. Furthermore, the focal zone, which is usually defined as the distance between the starting and ending axial points where the amplitude drops to $-6 \mathrm{~dB}$ of the amplitude at the focus, lies over a wide region when $a$ is decreased. Interestingly, there are no significant changes in pressure profiles for $a=40 \mathrm{~mm}$ and 50 $\mathrm{mm}$. This is because the critical angle $\theta_{c}$ for the $60 / 60$ $\mathrm{mm}$ lens is $32.5^{\circ}$ and an aperture radius greater than $32.2 \mathrm{~mm}$ does not in a sensitive way affect the field formation behind the lens.

Beam patterns on the focal lines are shown in Fig. 8 for the $60 / 60-\mathrm{mm}$ lens. The aperture radius is changed in the same way as in Fig. 7. The beam widens obviously when the aperture is decreased. For example, the total $-6-\mathrm{dB}$ beam width is $2.3 \mathrm{~mm}$ for $a=30 \mathrm{~mm}$ and almost the same as it is for the lens when $a=40 \mathrm{~mm}$ and $50 \mathrm{~mm}$. By contrast, the beam width becomes

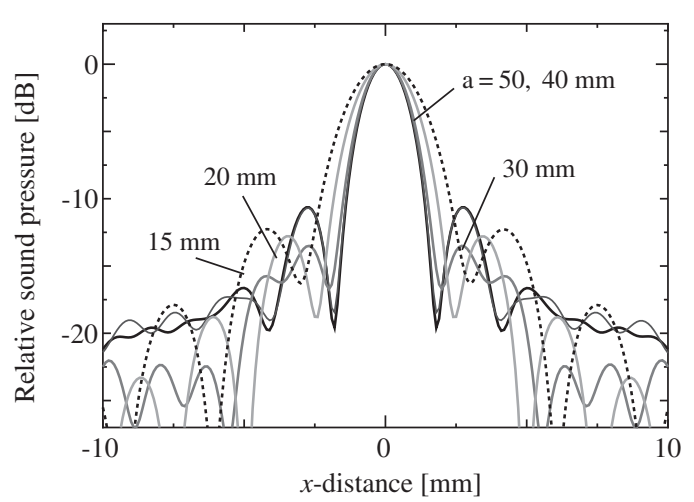

Fig. 8 Beam patterns for various aperture radii of the 60/60$\mathrm{mm}$ lens. Plane waves are normally incident on the lens. The receiving lines are chosen at the pressure peaks in Fig. 7; i.e., $z=56 \mathrm{~mm}$ for $a=50,40 \mathrm{~mm}$, $z=56.7 \mathrm{~mm}$ for $a=30 \mathrm{~mm}, z=58.3 \mathrm{~mm}$ for $a=20$ $\mathrm{mm}$, and $z^{\prime}=55.5 \mathrm{~mm}$ for $a=15 \mathrm{~mm}$. Sound absorptions in the lens are included in the data. 
somewhat broad at $2.9 \mathrm{~mm}$ for $a=20 \mathrm{~mm}$. As for the Rayleigh's criterion, $\Delta \theta$ at $a=30 \mathrm{~mm}$ has the same value, $1.4^{\circ}$, as in Fig. 6. However, the actual resolution is $1.7^{\circ}\left(=\tan ^{-1}(1.65 / 56.7)\right.$, slightly larger than the resolution of the 100/35.5-mm lens. Sidelobe pressure levels are relatively high for the lenses with the 40$\mathrm{mm}$ and larger apertures, probably due to the aberration. Of the five lenses, the $30-\mathrm{mm}$ radius lens has the lowest level in the first sidelobe. When the radius becomes small, the aberration is weakened, and the side-lobe levels are decreased. A reduction in levels of about 3-dB reduction is found at the first sidelobe by comparing the data of $a=40 \mathrm{~mm}$ and $30 \mathrm{~mm}$. Overall, the sidelobe levels range from $-10 \mathrm{~dB}$ to $-15 \mathrm{~dB}$. These levels are a few decibels higher than those of the corresponding spherically curved lens ${ }^{8)}$.

\section{Conclusions}

The present theoretical report has examined numerically the opinion that the generation of shear waves in an acoustic solid lens has no great effect on focused fields, even for relatively thick lenses with widely opening apertures that include the critical angle. The fields near the focus never change significantly their features. The result thus obtained definitely supports the validity of the previous presumption or opinion that the presence of shear waves, caused by mode conversion, is not important in predicting lens performance. This means that in place of the fluid/solid model presented here the fluid/fluid model that does not need to include the generation of shear waves is in principle acceptable for analytical predictions of lens performances. Consequent simplification of the theory and numerical computation will help considerably in the further development of ultrasonic lens design. Needless to say, it should be noted that wave diffraction due to a finite lens aperture becomes a major effective factor in beam focusing.

The present work is partially supported by the Min- istry of Education, Science, Sports and Culture, Grantin-Aid for Exploratory Research, 16656062, and by the Mitsubishi Electric Corporation.

\section{Appendix A}

Using eqs. $(6) \sim(8)$, the displacement components $u_{r}$ and $u_{\theta}$ are given by

$$
\begin{aligned}
u_{r} & =\frac{\partial \psi}{\partial r}+\frac{1}{r} \frac{\partial A_{y}}{\partial \theta} \\
& =\sum_{n=-\infty}^{\infty} a_{n}\left\{k_{l} B_{n} H_{n}^{(2)^{\prime}}\left(k_{l} r\right)-\frac{n^{2}}{r} C_{n} H_{n}^{(2)}\left(k_{t} r\right)\right\} e^{-j n \theta}
\end{aligned}
$$

$$
\begin{aligned}
u_{\theta} & =\frac{1}{r} \frac{\partial \psi}{\partial \theta}-\frac{\partial A_{y}}{\partial r} \\
& =-j \sum_{n=-\infty}^{\infty} n a_{n}\left\{\frac{B_{n}}{r} H_{n}^{(2)}\left(k_{l} r\right)-k_{t} C_{n} H_{n}^{(2)^{\prime}}\left(k_{t} r\right)\right\} e^{-j n \theta}
\end{aligned}
$$

Taking account of the boundary conditions described in the body, we obtain the following three equations:

$$
\begin{aligned}
& H_{n}^{(1)^{\prime}}\left(z_{1}\right) A_{n}+\frac{z_{2}}{z_{1}} H_{n}^{(2))^{\prime}}\left(z_{2}\right) B_{n}^{\prime} \\
& -\frac{n^{2}}{z_{1}} H_{n}^{(2)}\left(z_{3}\right) C_{n}^{\prime}=-H_{n}^{(2)^{\prime}}\left(z_{1}\right), \\
& H_{n}^{(1)}\left(z_{1}\right) A_{n}+\frac{1}{\rho c_{l}^{2}}\left\{\lambda H_{n}^{(2)}\left(z_{2}\right)-2 \mu H_{n}^{(2)^{\prime \prime}}\left(z_{2}\right)\right\} B_{n}^{\prime} \\
& -\frac{2 \mu n^{2}}{\rho z_{3}^{2} c_{t}^{2}}\left\{H_{n}^{(2)}\left(z_{3}\right)-z_{3} H_{n}^{(2)^{\prime}}\left(z_{3}\right)\right\} C_{n}^{\prime} \\
& =-H_{n}^{(2)}\left(z_{1}\right) \text {, } \\
& 2\left\{z_{2} H_{n}^{(2)^{\prime}}\left(z_{2}\right)-H_{n}^{(2)}\left(z_{2}\right)\right\} B_{n}^{\prime} \\
& +\left\{z_{3} H_{n}^{(2)^{\prime}}\left(z_{3}\right)-z_{3}^{2} H_{n}^{(2) "}\left(z_{3}\right)-n^{2} H_{n}^{(2)}\left(z_{3}\right)\right\} C_{n}^{\prime} \\
& =0 \text {, }
\end{aligned}
$$

where $z_{1}=k d, z_{2}=k_{l} d$, and, $z_{3}=k_{t} d$. Additionally, $B_{n}^{\prime}=j \omega B_{n}$ and $C_{n}^{\prime}=j \omega C_{n}$. The coefficients $A_{n}$ to $C_{n}$ are completely determined by solving eqs. (A.3) to (A.5), and the resultant solutions yield: 


$$
A_{n}=\frac{\beta_{1}\left(\alpha_{22} \alpha_{33}-\alpha_{23} \alpha_{32}\right)+\beta_{2}\left(\alpha_{13} \alpha_{32}-\alpha_{12} \alpha_{33}\right)}{\alpha_{11}\left(\alpha_{22} \alpha_{33}-\alpha_{23} \alpha_{32}\right)+\alpha_{21}\left(\alpha_{13} \alpha_{32}-\alpha_{12} \alpha_{33}\right)},
$$

$$
\begin{aligned}
B_{n} & =\frac{B_{n}^{\prime}}{j \omega}=-\frac{4}{\pi \omega z,} \\
& \times \frac{1}{\alpha_{11} \alpha_{22}-\alpha_{12} \alpha_{21}+\alpha_{32}\left(\alpha_{21} \alpha_{13}-\alpha_{11} \alpha_{23}\right) / \alpha_{33}},
\end{aligned}
$$

$$
C_{n}=\frac{C_{n}^{\prime}}{j \omega}=-\frac{\alpha_{32}}{\alpha_{33}} B_{n},
$$

where $\alpha_{11}=H_{n}^{(1)^{\prime}}\left(z_{1}\right), \alpha_{12}=\left(z_{2} / z_{1}\right) H_{n}^{(2) \prime}\left(z_{2}\right), \alpha_{13}$ $=-\left(n^{2} / z_{1}\right) H_{n}^{(2)}\left(z_{3}\right), \alpha_{21}=H_{n}^{(1)}\left(z_{1}\right), \alpha_{22}=\left\{\lambda H_{n}^{(2)}\left(z_{2}\right)\right.$ $\left.-2 \mu H_{n}^{(2) '}\left(z_{2}\right)\right\} / \rho c_{l}^{2}, \alpha_{23}=-2 \mu n^{2}\left\{H_{n}^{(2)}\left(z_{3}\right)-z_{3} H_{n}^{(2) '}\left(z_{3}\right)\right\}$ $/ \rho z_{3}^{2} c_{t}^{2}, \alpha_{32}=2\left\{z_{2} H_{n}^{(2) '}\left(z_{2}\right)-H_{n}^{(2)}\left(z_{2}\right)\right\}, \alpha_{33}=z_{3} H_{n}^{(2) '}\left(z_{3}\right)$ $-z_{3}^{2} H_{n}^{(2) "}{ }^{\prime \prime}\left(z_{3}\right)-n^{2} H_{n}^{(2)}\left(z_{3}\right), \beta_{1}=-H_{n}^{(2) '}\left(z_{1}\right)$, and $\beta_{2}$ $=-H_{n}^{(2)}\left(z_{1}\right)$.

\section{References}

1) D. L. Folds, "Focusing properties of a solid ultrasonic cylindrical lenses," J. Acoust. Soc. Am., 53, 826-834 (1973).

2) D. L. Folds and J. Hanlin, "Focusing properties of a solid four-element ultrasonic lens," J. Acoust. Soc. Am., 58, $72-77$ (1975).

3) H. W. Jones and C. J. Williams, "Lenses and ultrasonic imaging," in Acoustical Holography, edited, L. W. Kesseler Eds. (Plenum, New York, 1977), 133-153.

4) H. W. Jones and H. W. Kwan, "Ultrasonic lenses for imaging," Ultrasonics, 23, 63-70 (1985).

5) K. Fink, "Computer simulation of pressure fields generated by acoustic lens beamformers," Master thesis at the University of Washington, 1994.

6) T. Anada, T. Tsuchiya, N. Endoh, and T. Nakamura, "Numerical analysisi of underwater acoustic lens using wide-angle parabolic equation method," Jpn. J. Appl. Phys., 41, 3509-3512 (2002).

7) K. Mori, T. Nakamura, T. Yokoyama, and A. Hasegawa, "3-d FDTD analysis of sound field focused by biconcave acoustic lens for normal incidence," Jpn. J. Appl. Phys., 44, 4696-4701 (2005).

8) T. Kamakura, K. Aoki, and T. Nakamura, "Focusing ultrasonic waves by a bi-concave lens: normal incidence on the lens," Submitted to acta acustica united with Acustica.

9) M. Akiyama, H. Uchiyama, and T. Kamakura, "Focused sound beams by an elliptic surface lens," J. Acoust. Soc. Jpn., 61, 71-78 (2005).

10) B. Hartmann and J. Jarzynski, "Ultrasonic hysteresis absorption in polymers," J. Appl. Phys., 43, 4304-4312 (1972).

11) E. Juliac, J. Arman, and D. Harran, "Ultrasonic interferences in polymer plates," J. Acoust. Soc. Am., 104, 1232-1241 (1998).

12) Y. Bouzidi and D. R. Schmitt, "A large ultrasonic bounded acoustic pulse transducer for acoustic transmission goniometry: Modeling and calibration," J. Acoust. Soc. Am., 119, 54-64 (2006).

13) W. C. Elmore and M. A. Heald, Physics of Waves (Dover Publications, New York, 1985), Sec. 10.4. 